\title{
Functional Role of Colour in Preattentive Cognitive Processing from Thematic Map Images
}

\author{
Vineeta John \\ Head, Geography Department, Ewing Christian College (An autonomous constituent College of Allahabad \\ University ALLAHABAD
}

\begin{abstract}
The growing use of colour in thematic map design and production, particularly in GIS based mapping environments, brings to notice a state of affairs where at least certain basic rules are required to be made to help in better, effective and efficient use of colour. One approach towards this end appears in efforts towards making the map image and input from it more compatible with the human visual processing system. This entails selection and structuring of the visual variables and the ensuing sign system of map graphics into a map image in a way in which such structuring results in an increase in the efficiency of information processing system. Many aspects of use of these visual variables from the commonly used term colour i.e. hue, value and chroma in symbolisation of thematic maps have not been investigated individually in the areas as segregation of map image's focal realm form its peripheral realm or in other words the figure from its ground, organising perceptual groups, creating visual hierarchies by depth cues. The present paper attempts to fill certain gaps in studies related to the effects of the use of visual variables hue, value and chroma individually or in combination in map symbolisation on the preattentive stage of visual processing of information. These effects will be studied in case of area symbolised and line symbolised maps including those graduated point symbolised maps where colour dimensions are used as area fills in the blank outline symbol forms. Evidence in support of these effects will be sought from the experimental studies in design research in thematic cartography and psychology.
\end{abstract}

Keywords: Colour Dimensions, Colour Use, Visual Information Processing, Preattentive Processing, Chroma, Hue, Value, Figure ground, Hierarchical Organisation, Visual Organisation

\section{Introduction}

Increasing use of colour in map design and production, particularly in GIS based mapping environments, where mapping is mostly done by persons not trained in cartography or novices in cartographic practice, points to a situation where at least certain basic rules are required to be made to help in better, effective and efficient use of colour. One approach towards this end appears in efforts towards making the map image and input from it more compatible with the human visual processing system.

One of the first steps in such an approach is to distinguish the manner of input of visual information from such sources as thematic maps, which unlike the case of textual displays, does not follow a definite sequence. In case of maps the receptors can and do start the acquisition of input from any part of the image and can stop at any point (Robinson and Petchenik, 1976 [1]). These observations can be confirmed by several eye movement recordings of map percipients (Jenks [2]). Processing of information from map displays is also different from those of discursive propositional symbolism (Peterson, 1987 [3]). This distinction has been elaborated by Robinson and Petchenik (1976: 44-45 [1]). They have pointed that in case of discursive symbolic displays of languages i.e. texts, the intended meaning is processed and apprehended from a linear series of visual fixations over different symbols arranged in a linear sequence. But in case of displays like maps this intended meaning is carried by a whole figure that consists of a complex array of interacting sub-figures. The cognitive system receives the input from such sources in a variable unsystematic and non-linear sequence of fixations. It is processed as a whole through the relations of component parts within the total structure. In addition, the processing of the input from maps, as compared to textual displays, involves association of one visual stimulus to another in a relational context of a system of visual signs that do not have fixed meanings rather than to relations of a visual stimulus from signs belonging to a system of invariant sounds and meaning.

These characteristics of information input and its processing from thematic maps thus point to the necessity of simultaneous consideration of the characteristics of the percipient and the map towards understanding and meeting the requirements of effectiveness, efficiency and usability in map image structure. These considerations lead to the concepts of compatibility developed in human factor engineering or engineering psychology which is a field concerned with the man / machine interactions and how well these two go together along with the nature of human cognitive capacities. Such concerns produced the questions about the best configurations for desired output patterns. Answers to these questions led to the notion of compatibility between the input and the human cognitive system for optimal performance (Fitts and Seeger, 1953 [4]; 
Lachman et. al. 1979 [5]) and allocation of preattentive and attentional resources between different tasks. These concerns are based on the notion that design of the maps like the design and operation of machines must take advantage of human information processing potential and must be able to accommodate with the limitations of human percipient/operator.

Thus these notions can be extended to improving effectiveness and efficiency of visualisation from maps under the logic that what is considered essential for man / machine interaction must be equally applicable for man / map interaction in visualisation as well in communication situations. Because, both machines and maps are artificial creations and in both cases the optimal performance is based on human information processing potential. This notion, in the present context, may not be necessarily limited to a fit between different stimuli and their response patterns but, may be extended to cover the internal cognitive translations that intervene between input and human behavioural responses. Some cartographers have made passing references to such questions in improving visualisation and communication capability of maps (Petchenik, 1983 [6]; Castner 1983 [7]). While Robinson and Petchenik [1], in this connection, have emphasised the need of taking into account the functioning of the human visual cognitive system "in predicting the potential of the map percipient for comprehending *or visualisations from the map" (1976: p. 68 [1]). Wood (1972 [8]) and Board and Taylor (1976 [9]) support this view and have examined the role of human factors in map design and communication (*italics added). A more recent work in this area is that of Eastman (1985 [10]).

This entails selection and structuring of the visual variables and the ensuing sign system of map graphics into a map image in a way in which such structuring results in an increase in the efficiency of information processing system. This increase can be obtained by ensuring availability, and a better quality in available information, from the map image as an input to the processing system. These objectives may be met through selection of the basic elements of the sign system during the map's image structuring. These modulations are required to be made in view of the behaviour of the system in terms of the requirements of its functional components, functioning and organisation of the processes, and the operations of the mechanisms of these processes. Colour and its three visual variables are such promising visual variables in this respect. Their importance as visual variables in mapping has increased many folds with the present developments in the easily available computers and related colour related printing accessories together with the availability of mapping and GIS software.

Many aspects of use of these visual variables from the commonly used term colour i.e. hue, value and chroma in symbolisation of thematic maps have not been investigated individually in the areas such as segregation of map image's focal realm form its peripheral realm or in other words the figure from its ground, organising perceptual groups, creating visual hierarchies by depth cues (Carr, D. 1994 [11]).

\section{Objective}

The present paper aims to fill certain gaps in studies related to the effects of the use of visual variables hue, value and chroma individually or in combination in map symbolisation on the preattentive stage of visual processing of information. These effects will be studied in case of area symbolised and line symbolised maps including those graduated point symbolised maps where colour dimensions are used as area fills in the blank symbol outline forms. Evidence in support of these effects will be sought from the experimental studies in design research in thematic cartography and psychology.

\section{Colour As Visual Variable}

Colour, in its extended applications in thematic maps, appears to have a large potential. The expectations of this potential arise due to the unitary nature of a colour patch. This is because of the primacy and spontaneity of colour experience in any visual perception. The visual appearances of forms are processed first in terms of the hue, value and chroma information where present. The outlines that define a two dimensional shape are basically established by the differences in hue and brightness i.e. a combination of the colour dimensions of value and chroma. Similar information is also responsible for the perception of three-dimensional forms (Arnheim, 1969 [12]). The spontaneity of the experience can be explained in terms of the available experimental evidence (Rensink and Enns, 1995 [13]), which suggests preemption by colour information in visual processing. In addition, the use of different colour attributes in redundant combinations for symbolisation has been suggested by Schlichtman (1979 [14]) to improve the processing of information from maps. This suggestion has support in a sizable body of published research in psychology.

\section{Human Visual Information Processing}

The structuring of a map image, which meets the efficiency and usability requirements of a visualisation tool and medium of communication necessitates a consideration of the map percipient as an active processor of the information. This consideration derives its importance from the more specific nature of map displays that puts them apart from other visual information sources and even from other symbolic displays. The 
nature of this symbolism, which takes the form of graphic map images, imposes particular constraints, specially, on the manner of its processing and storage

Developments in the field of cognitive psychology have shown that the human beings act as active information processors and a perceptual output is not produced as an instantaneous response to a stimulus. It is obtained as a result of processing the input in a different stages through a system of functional components. These cognitive mechanisms and processes, in addition to creating new knowledge, may also be involved in its utilization and communication from many sources including thematic maps, which form a very important visual medium of information from the milieu around us. The thematic maps are the primary images of some of the aspects of the spatial milieu because such aspects of the milieu are intangible and besides being intangible generally extend beyond the human sensory limits.

These map images use visual forms of symbolism in which the parts constituting the image are unlike visual world around us. These map images use visual forms of symbolism in which the parts constituting the image unlike languages, are presented simultaneously and not successively. In addition, the meanings of the component elements of such images are seen to be comprehended and conveyed in terms of the whole and through their interrelations within the total structure of an image (Langer 1951: 86-89 [15]).

\subsection{Processes of the Human Cognitive System}

Currently held views model the processes of the visual cognitive system, following Neisser (1967 [16]), in a two-stage hierarchical architecture. (Treisman, A. and Gelade, G. 1980 [17]). The model makes an attempt to account for the functions of both the stored knowledge and the stimulus information in visual information processing. It tries to accommodate the role of local features and global properties, which represent the relationships between the component parts and the whole. It also makes an effort to reconcile the conflicting experimental evidence related to the use of either sequential and serial or spatially and operationally parallel mode of processing in cognitive activities. The two envisaged hierarchical stages in the operation of these processes have been named as 'preattentive' and 'focal attentive'.

The initial level of visual cognition, the 'preattentive' stage of processes, which involves basic features, operates in parallel (Treisman, A.1985 [18]). Preattentive processes operate on the input to the system held briefly in iconic delay. The input is pre-processed to some extent and is transformed into symbolic representations. This initiates the central processes and is considered the first phase of central representations arising out of these processes.

The processes at the second level are seen to be characterised by a more active involvement of the higher order central processes. These central process, here are taken to mean the functions which bring about an interaction between input data and stored knowledge on the one hand and on the other with such factors as expectations and preferences. The depth and course of these processes are in part contingent upon the input data and are partly dependent on the extra-input information from stored schemata and the factors of expectation and preference.

\section{Preattentive Processes}

Wolfe and Horowitz (2008 [19]), in respect of these initial preattentive processes point out that:

"The original use of preattentive had a spatial/neural aspect to it, implying that some brain loci were preattentive. More modern understandings suggest that an area like primary visual cortex might initially process a visual stimulus without showing an influence of attention. However, activity in the very same piece of cortex might be subsequently modulated by attention in a reentrant manner (Di Lollo, Enns, \& Rensink, 2000 [20]; Lamme \& Roelfsema, 2000 [21]; Saalmann, Pigarev, \& Vidyasagar, 2007 [22]). The most helpful use of the term preattentive is a temporal usage. Prior to deployment of attention to an object, any visual processing of that object is, by definition, preattentive."

These processes construct an integrated view of a continuous scene, such as the one obtained from a map image from a succession of representations. But dealing with such a large amount of input for its cognitive processing will require too large a processing capacity or too much previous experience. The processes of the first level 'segment' the input into the figure and ground components to produce what Hebb (1949 [23]) called 'primitive unity'. The segmented input is then segregated into involved figural units, which are also simultaneously organised into ordered chunks. This preattentively processed input is used by the later processes of human cognition. This kind of processing of the input is required to be carried out in parallel over the scene or image space and simultaneously over all feature dimensions. Neisser's formulations prescribed that these operations must be truly 'global' or 'holistic' and each element in the visual field must entirely be segregated from others. They also must take place very early in the processing and be very rapid, effortless and automatic. Sufficient experimental evidence exists for such modelling. Representative examples for such evidence of parallel operations can be cited in the works of Arguin and Cavanagh (1988) [24], Lindsay and Norman (1972 [25]). These operations are carried out in the earliest milliseconds of viewing a visual display (Palmer, 1975 [26] 
and are very rapid. They occur within a few hundred milliseconds. The operations are performed without any effort and are automatic (Logan, 1992 [27]). In Neisser's views, as compared to processes of the second level, the preattentive processes are crude and are error prone.

According to Neisser's formulations, the preattentive processes, in the initial phases, are seen to operate on the sensory properties for segmentation of the input. Beller (1970 [28]) while testing Neisser's two stage model of visual information processing, obtained experimental evidence of the use of easily discriminable physical properties in such operations. Rensink and Enns (1995 [13]) describe these properties as being simple and belonging to a set of 'visual primitives'. Thus the initial segmentation by these processes may be taken to be based on those visual primitives that produce heterogeneity in the visual field (Wever, 1927 [29]; Forgus, 1966 [30]; Dent, 1972 [31]). These properties include luminosity, wavelength composition, texture, spatial extent and any bounding mechanism in the form of a contour.

Recent evidence from an experimental study of Bravo and Blake (1990 [32]) suggests that these processes may not be limited to the use of only simple input properties in these operations. This suggests an expanded role for this level of operations in human visual information processing. Neisser (1967:97 [16]) also suggests a constructive role for these processes in the segregation and the subsequent grouping of the input. In this view, these processes in very limited operations are seen to organise chunks out of the input which have already gone through the initial phases of this level of processing. These constructed chunks take the form of figural units and sub-figures. Such chunking or groupings by necessity are a complementary process of segregation (Arnheim 1969:70 [12]; Olson and Attneave 1970:1 [33]). Such chunks are defined by their higher order figural characteristics. The global level relations which connect symbols into symbol structures or chunks in the two dimensional space are mainly the relations of similarity both phenomenal and spatial. Inclusiveness and continuity are among other relations that can produce such groupings.

However, this constructive segregation is not limited to the two-dimensional space only but extends along the third dimension too. In such cases the organization segregates these chunks in hierarchically arranged vertical planes along the dimension of depth. This type of hierarchical organization in the visual field is produced by the relations of texture and density gradients, surface contrast gradients including gradients of colour. The unambiguity in such segregations is produced by the stabilization of the separated units, which in turn depends on the quality, and availability of information from the input. Though, these early processes are seen by Neisser as limited in function; they are supposed to 'delineate units' which yield global properties and provide partial cues to guide shifts of attention. Wolfe (1992 [34]) and Wolfe and co-workers (1990 [35]) see the specific role of these processes in guiding the later serial processes in the second stage in selecting the elements of interest in the visual field for further processing. The extent of these functions is limited to the immediate present and to partial analysis of the input.

Modelling the system in terms of the operations performed by its functional components suggest that features in the icon are the initial representations of the input. The availability of the feature dimensions and their quality in these representations, to a large extent, depends on the nature of sensory data available from the map image and the effectiveness of the pre-processing of the input from the map. These factors, in turn, determine the effectiveness of the subsequent phases in the continuum of preattentive processing. This efficiency plays a crucial role in the information processing from maps because it is the stage where the system has to deal with the greatest load of information in the form of almost raw data. The processes, of the preattentive stage, have to perform operations which segment, segregate as well organise the input. These processes are also required to monitor the structured input from the map display for its important aspects, which are then used by the subsequent higher-level processes in the current context.

\subsection{Mechanism of the Preattentive Processes}

The processes of the system operate through two complementary mechanisms that integrate the two levels of the processes. In addition, they also simultaneously serve as control schemes for the processing. These are known as 'bottom-up' and 'top-down' mechanisms. The primary evidence for the existence of such mechanisms comes from the study of eye movements. The patterns of these movements, though being highly individualistic in themselves, can be taken as the macro-level manifestations of the processing activity (Eastman, 1985 [10]). Eye movement recordings of the subjects looking at displays during information processing and search patterns obtained from these recordings are indicative of the operation of such mechanisms.

Bottom-up mechanisms relate to preattentive processing and are set into operation by the incoming sensory data. The processing proceeds in smooth logical progression of different stages. The output from each stage, in this sequence, is taken to serve as the input data that drives the next stage. The processing by these mechanisms is also described as data-driven or stimulus-driven. Bottom-up processing mechanisms operate automatically being triggered by the arrival of new information and require almost no amount of attentional resources for processing (Lindsay and Norman, 1972:347 [25]). 
It is the preattentive stage in the processing where the need of compatibility for system efficiency improvement is most strongly felt. At the same time it is here that the system responds markedly to systematic efforts in building the compatibility through deliberate and planned map design efforts. This contention has support in Phillips (1984 [36]) and Gill's (1988 [37]) results who found a greater effect of map design improvement on the initial preattentive stage of processing as evidenced in the improvements in the subjects' performance.

\subsection{Figure-ground Segmentation}

Starting with the initial mode of the input to the system where the retinal projections are internalised, there is evidence that these projections are pre-processed to a limited degree (Haber and Hershenson, 1973 [38]). The mechanism involved here are the lateral and spectral inhibition (Hurvich and Jameson, 1974 [39]) which respectively produce edge enhancement effect and simultaneous contrast effect. The extent to which this input is pre-processed during its transformation phase depends on the availability of information from the map. The optimisation of this pre-processing, thus, will depend on the selection of map image elements and their constituents and their modulations while structuring the corporeal map image. One of the modulators, required for edge enhancement is the degree of contrast between the surfaces of different map image elements. This contrast makes either of the sides of a form dominant or more articulated for an edge to be seen. In case of simultaneous contrast effect the modulators take the form of prominent contours (Bermen and Leibowitz, 1965 [40]) or of dividing lines to separate two stimuli (Gilmartin, 1981 [41]).

The subsequent functioning of the processes of the modelled system demands segmentation of the initial representations from a map image into figure and ground components during the earliest phases of the processing. Effective segmentation in the map image space, in these early phases, depends on the availability of information, which produces differentiation by separateness of the forms through continuous contours and edges and by the togetherness of their features. In addition, it also requires the presence of those feature dimensions that create heterogeneity in the visual field and leads to a protrusion of the figural fields. Dimensions of the stimulus which produce such heterogeneity are hue differences, differences of value and chroma, commonly described as brightness (Luke, 1996: 115 [42]) and differences in lower level shape features (Wever, 1927 [29]; Arnheim, 1969 [12]).

\subsection{Visual Organisation}

Once the input has been segmented in the initial phases, global level analysers operating in parallel all over the image space organise the input for its focal attentive processing. This is also a requirement of the working memory unit in view of its spatial capacity constraints. This organisation entails the grouping of the segmented input into larger units irrespective of their location through the structural links of relations. Such relations are defined by the principle of similarity. "This principle asserts that the degree to which parts of a pattern resemble each other in some perceptual quality will help determine the degree to which they are seen as belonging together" (Arnheim, 1969: 67 [12]). In case of the thematic maps too, similarity relations are obtained from the sensory information in the form of global properties. These properties are made available to the system from the graphic variables used in characterisation of attributes in the map image by the bottom up mechanisms. These relations of similarity are required to be so strong as to overcome the effects of spatial separations and influence of proximity.

\subsection{Organisation of Visual Hierarchies}

The groups so defined are also required to be simultaneously organised in a schematic hierarchy. The position of objects in such a hierarchy is a function of those feature dimensions, which among other things, produce relations of order along the dimension of depth or distance in the three dimensional space. Such relations are based on the information, in the form of secondary spatial cues, available from those feature dimensions, which produce perceptual gradients. A gradient in this context has been defined as "an increase or decrease of something along a given axis or dimension" (Gibson, 1950 [43]). These gradients can be of edges, surfaces, texture, colour, and of sizes. Experimental evidence from map use studies suggest that such schematic hierarchies are latter required in relating these chunks into the underlying multilevel constituent structure of the map images for comprehension of displayed spatial information, through which this information is stored and recalled (Thorndyke and Stasz, 1980 [44]; Eastman, 1985 [10]).

The next stage of processing, which follows instantly, is seen to use these organisations and the global properties defining such organisations in selective guidance and allocation of focal attentive operations. In case of graphic displays like maps, such selectivity is likely to be based on representations which can be thought to be produced, during the preattentive processing, by those graphic characteristics of the map image elements which visually organise these into separate visual levels with different degree of figural prominence. This requirement can also be met by transformation of referent content into graphic form through the modulations of 
graphic variables. Such graphic encoding establishes a syntax, which is embedded in the form itself by its definition. The embedded syntax organises a relationships between the thematic symbols of the graphic statement of a map and at the same time establishes a sequence of order in the visual field. The employed graphic variables should posses those visual dimensions that not only characterise but also modulate the amplitudes of the required relationships of similarity, separateness and of order. Such organisation of the relations and the acquired sequence of order in the global properties of the map image partly provide a basis for the selectivity in the allocation of focal attention.

\section{Functional Role Of Colour}

The sensation of colour defined in terms of its components viz. Hue, Value and Chroma in Munsell's (1991 [45]) terminology have a marked influence on visual processing from an image where these are used as elements of the image. The same is the case with the thematic map images where colour sensation components are used as visual variables in map symbolisation. The following sections will examine the functional role of colour at different stages of preattentive processing of information from map images.

\subsection{Input Segmentation}

The change in the character of the surface of the map symbols relating to the appearance of the surface i.e. to their colour dimensions produces a primary heterogeneity in the map image particularly in its focal realm. It is supposed to create a primitive segmentation between these symbols and the elements of locational component together with the otherwise homogeneous white plane of the substrate. The manner, in which the change in surface character is brought about, creates a visual barrier or resistance for the eyes to penetrate. This visual resistance, in a way, provides areal extent as well micro structure to the map symbols (Katz. 1958 [46], Koffka 1935 [47]). In addition, it is expected to give, in Rubin's (1958 [48]) words, a 'thing character' to hitherto undifferentiated open map forms. The information in the symbol forms, so far limited to contours only, is in this way expected to be reinforced in the very early phases of the process of segmentation.

The change in surface character makes the area enclosed by the map perimeter more dominant. The effects of this kind of surface appearance extend to create marked brightness differences in the visual field. The dominance of one of the sides causes an edge to appear and allows a definite contour to develop. This heterogeneity is also known to create sharp edge gradients, which result in the definite localisation of figures in depth and to their stabilisation. These expectancies have support in the experimental evidence obtained by Wever (1927 : 217-218 [29]) which shows that a large difference in luminous reflectance between a relatively dark homogeneous figure having a surface character and an equally homogeneous white ground is required for a 'perfect' figure-ground experience and it is in addition to the requirement of a sharp and continuous contour. Oyama (1960 [49]) too has come to a similar conclusion for the necessity of a large value contrast between the figure and ground for the stabilisation of figure. Lindauer and Lindauer (1970 [50]), using the Rubin's face and vase image with a control stimulus of only black outline, have also shown that the number of responses for the filled area as figure had increased with the increase in the contrast between the two areas. In case of maps similar conclusions were drawn by Wood (1968 [51]) and later by Spiess (1978 [52]) that the segmentation of different shapes as figures is mainly a function of the degree of contrast in the two fields of the map. McCleary's (1981 [53]) results similarly lay stress on the importance of the role of value variations in creating heterogeneity and articulation in the different visual fields of a map for the separation of different shapes as figures. The effects of brightness or value variations have also been stressed by MacEachren and Mistrick (1992 [54]) in case of achromatic thematic maps. Their results can easily be extended in case of chromatic maps too.

The stability in the segmented figures has similarly been noticed to be further strengthened by the surface texture created homogeneity: a thing experience in the figural areas. Wever (1927: 217, 223 [29]) found that the surface texture provides a difference in the depth localisation of figure and ground. Dent (1972:88 [31]) too has experimentally demonstrated the importance of the role of texture differences in the development and localisation of figure and ground surface in maps. Such results were also obtained in a relatively recent study in cartography related to this stage in information processing from thematic maps ( McCleary 1981 [53]). The experimental evidence reviewed so far leads to very strong expectations that the changes in surface appearance of symbols produced due to the use of colour variables will increase the ease i.e. the speed with which the input from the map image is segmented into figural units. The combined effect of the various cues available due to the use of different colour variables further ensures the rapid stabilisation of the segmented figures. In this way the changes are expected to eliminate the ambiguous situations and in turn end the multistability in the perception of thematic input from the map at this stage.

\subsection{Visual Organisation of the Input}

The application of the colour dimensions as area symbols, in line symbols, or as area fills to the black outlines of graduated point symbols lends a microstructure to these symbols. This obtained surface character, in 
addition, tends to produce a kind of internal unity in the plan forms of the map symbols. The unity provides information on areal extents of the maps and is expected to produce an improvement in the quality of information made available to the earliest processes of the system (Wood 1972 [8]). Moreover, the presence of different variables of colour individually or in combinations particularly hues, adds to the discriminability of the symbols (Keates 1982 [55]). This effect is ascribed to their functional role as visual primitives (Melara et.al. 1993 [56]). In view of the manner of the modulation of the colour variables in symbolisation some of the colour variables may selectively supplement the information by providing better discriminability in the class and size ranges where larger JNDs are required for discrimination. This additional information can be expected to overcome the limitations of size and class difference based discriminability of both graduated point or line symbols and area symbols. Experimental evidence in support of the expectancies for increased discriminability can be seen in the early work of Erikson and Hake (1955 [57]), observations of Arnheim (1969: 323 [12]) and Mersey's (1990 [58]) work in cartography. In case of the supplementing role of some of the colour dimension vis-à-vis their role in increasing the discriminability reliance can be placed on the empirical discrimination functions derived by Munsell (1991 [45]) in case of 'value' and the extension of these functions to 'hues' ordered in partial spectral progression on the basis of their inherent luminosity differences. This enhanced discriminability may also reduce the possibilities of tentative size and class based differentiations and as a result may also eliminate the chances of ambiguities in the bidimensional chunking of map symbols in the map image.

The enhancement in relative discriminability throughout the class and size range of map symbols, both in area, line and graduated point symbolisations, is seen to allow the global level analysers, operating in parallel all over the map image space, to make efficient and unambiguous selections and to construct chunks out of the segregated input. This selectivity is expected to be both effective and faster because of the availability of additional and better similarity information from the primitive properties of the different dimensions of colour. The additional information provided by the primitive visual properties of colour dimensions is seen to support the selection and subsequent grouping engendered by similarities in the focal realm of map's space (Arnheim 1969: 73 [12]).

This support is further seen to strengthen the structural links of similarity relations among the units of segregated input and to group these units into larger chunks irrespective of their location. Such groupings are expected to be so strong or compelling as to overcome the effects of both locational separations and proximity due to the availability of this additional similarity information (Arnheim 1969: 73 [12]). In this way the possibilities of the emergence of alternate groupings of different sized map symbols on the basis of locational proximity or locational separations are almost eliminated. Thus the chances of ambiguities arising in such chunkings, as compared to conventionally symbolised achromatic maps are expected to be almost eliminated.

Such expectancies can further be examined in case of each dimension of colour separately. Hues, in their envisaged functional role, are expected to add to the size and class based selectivity of map symbols because of their separateness and mutual exclusiveness (Arnhiem (1969: 328 [12]). This separability in hues is seen to be enhanced further when the distinctions created by their inherent luminosity are taken into account. In this way hues are expected to have the most powerful effect on chunking of symbols in the map image space.

The role of value in selection and grouping may similarly be seen in adding to the size and class dependent differentiation and selectivity leading to efficient chunking of the segregated input. This becomes possible due to the availability of faster information on the dimension of value, which leads to better symbol definitions. When combined with hue this selectivity is further increased because of the human system's quick response to combined changes in these two dimensions (Arnhiem 1969 [12]).

Chroma, likewise, separately or in combination with other dimensions, particularly with value, (Melara et. al. 1993) is seen to subserve both segregation of symbols and their resultant groupings on the basis of the similarity relations. Experimental evidence relating to early and simultaneous processing along at least two feature dimensions together with evidence of faster selections in the presence of colour dimensions can be seen in the works of Wurm et. al. (1993 [59]), Yantis (1990 [60]), Arguin et.al. (1988 [24]), Robinson et. al (1984 [61]), Ward (1983 [62]), Imai and Garner (1965 [63]).

\subsection{Hierarchical Organisation}

The expected improvements in the quality of size and class information together with the additional and uniform discriminability in the symbols are likely to increase the effectiveness of the visual gradients engendered by the gradual increase or decrease of size and class based difference of map symbols. Such gradients, in turn, are expected to provide the required information to the processes operating at this stage for the simultaneous organisation of the segregated chunks of map symbols in the dimension of depth or distance through the relations of order. 
Troscianko and co-workers (1991 [64]) have experimentally demonstrated gradients of chroma e.g. red to gray to be particularly effective as a monocular depth cue. Chroma variations have been found to create depth effects quite efficiently in another way i.e. through 'aerial perspective' which adds to the effects of size and class variation based gradients. This perspective, first described by Leonardo da Vinci, is said to be produced by a 'faded' look evident because of refraction through an increasing body of air. A similar effect is also seen in conditions of fog when farther objects look more and more gray (Dent 1972: 83 [31]). According to Arnhiem (1969: 269 [12]) this effect can be made available effectively at small distances also because this effect too produces a perceptual gradient artificially.

\section{References}

[1]. Robinson, A. H., Petchenick, Barbara B. (1976) Seeing and Mapping, in Nature of Maps: Essays towards Understanding the Nature of Maps and Mapping, University of Chicago Press.

[2]. Jenks, G. F. (1973) Visual Integration in Thematic Mapping: Fact or Fiction, International Yearbook of Cartography 13, $27-35$.

[3]. Peterson, Michael P. (1987) The Mental Image in cartographic Communication, Cartographic Journal, 24:1, 35-41.

[4]. Fitts, P. M., Seeger, C. M. (1953) S-R Compatibility: Spatial Characteristics of Stimulus and Response Codes, Journal of Experimental Psychology, 46, 199-210.

[5]. Lachman, R., Lachman, J. L., Butterfield, E. C. (1979) Cognitive Psychology and Information Processing: An Introduction, Erlbaum. Hilsdale, N. J.

[6]. Petchenik, B. B. (1983) A Map Marker's Perspective on Map Design Research 1950-1980 In Graphic communication and Design in Contemporary Cartography, Taylor D.R.F.,37-68,Wiley, New York.

[7]. Castner, H. W. (1983) Research Questions and Cartographic Design, in Graphic Communication and Design in Contemporary Cartography, D. R. F. Taylor (Eds.) John Wiley, New York, 87-113 London.

[8]. Wood, M. (1972) Human Factors in Cartographic Communication, Cartographic Journal, 9, 123-132.

[9]. Board, C., Taylor, R. M. (1976) Perception and Maps: Human Factors in Map Design and Interpretation, Transactions Institute of British Geographers, 19-36.

[10]. Eastman, J. R. (1985) Cognitive Models and Cartographic Design Research, Cartographic Journal,22:2, 95-101.

[11]. Carr Dan (1994) Colour Perception, the Importance of Gray and Residuals, on a Choropleth Map, Statistical Computing \& Statistical Graphics Newsletter, vol. 2, 16-2

[12]. Arnheim R. (1969) Art and Visual Perception 'a psychology of the creative eye' Faber and Faber, London

[13]. Rensink, R.A., Enns, J. T. (1995) Preemption Effects in Visual Search: Evidence for Low-Level Grouping, Psychological Review, 102:1, 101-103.

[14]. Schlichtman, H. (1979) Codes in Map Communication, Canadian Cartographer.16,81-97.

[15]. Langer, S. K. (1951) Philosophy in a New Key, New American Library, New York

[16]. Neisser, U. (1967) Cognitive Psychology, Appleton Century-Crofts, New York.

[17]. Treisman, A., \& Gelade, G. (1980). A Feature-Integration Theory of Attention, Cognitive Psychology, 12, 97-136

[18]. Treisman A. (1985) Preattentive processing in vision, computer vision, Graphics and Image Processing 31, $156-177$.

[19]. Wolfe, J. and Horowitz, T. S. (2008), Visual search, Scholarpedia, 3(7):3325

[20]. Di Lollo, V., Enns, J. T., \& Rensink, R. A. (2000) Competition for Consciousness among Visual Events: the Psychophysics of Reentrant Visual pathways. Journal of Experimental Psychology: General, 129(3), 481-507.

[21]. Lamme, V. A., \& Roelfsema, P. R. (2000). The Distinct Modes of Vision Offered by Feed Forward and Recurrent Processing. Trends Neurosci., 23(11), 571-579

[22]. Saalmann, Y. B., Pigarev, I. N., \& Vidyasagar, T. R. (2007). Neural Mechanisms of Visual Attention: How Top Down Feedback Highlights Significant Locations, Science

[23]. Hebb, D. O. (1949) The Organization of Behaviour Wiley, New York, Quoted in R. H. Forgus 1966, McGraw-Hill, New York.

[24]. Arguin, Martin, Cavanagh, Patrick (1988) Parallel Processing of Two Disjunctive Targets, Perception and Psychophysics, 44:1, 2230 .

[25]. Lindsay, P. H. , Norman, D. A. (1972) Human Information Processing Academic Press, New York.

[26]. Palmer, S. E. (1975) Visual Perception and World Knowledge: Notes on a Model of Sensory-cognitive Interaction, in Explorations in Cognition, D. A. Norman, D. E. Rumelhart (Ed) San Francisco: W.H. Preeman, 279-307.

[27]. Logan, Gordon D. (1992) Attention and Preattention in theories of automaticity, American Journal of Psychology, 105:2, 317-339.

[28]. Beller, H. K.(1970) Parallel and Serial Stages in Matching, Journal of Experimental Psychology, 84:2, 213-219.

[29]. Wever, E. (1927) Figure and Ground in the Visual Perception of Form, 38, 194-226 American Journal of Psychology, 38, 194-226.

[30]. Forgus, R. H. (1966) Perception: The Basic Process in Cognitive Development, McGraw - Hill, New York.

[31]. Dent, B. D. (1972) Visual Organization and Thematic Map Communication, Annals, Association of American Geographer, 62, 7993.

[32]. Bravo, M., Blake, R. (1990) Preattentive Vision and Perceptual Groups, Perception, 19:4 515-522.

[33]. Olson, R. K., Attneave, F. (1970) What Variables Produce Similarity Grouping, American Journal of Psychology, 83, 1-21.

[34]. Wolfe, Jeremy M. (1992) The Parallel Guidance of Visual Attention, Current Directions in Psychological Science, 1:4, 124-128.

[35]. Wolfe J. M., Stewart M. I. and Hill, et. al. (1990) Limitation on the Parallel Guidance of Visual Search: Colour x Colour and Orientation x Orientation Conjunctions, Journal of Experimental Psychology: Human Perception and Performance 16:4, 879-892.

[36]. Phillips, R. J. (1984) Experimental Method in Cartographic Communication Research on Relief Maps, Cartographica, 21:1, 120128.

[37]. Gill, Graham A. (1988) Experiments in the Ordered Perception of Coloured Cartographic Line Symbols, Cartographica, 25:4, 3649.

[38]. Haber, R. N. and Hershenson, M. (1973) The Psychology of Visual Perception Holt, Rinehart \& Winston, New York

[39]. Hurvich, L. M. and Jameson, D. (1974) Opponent Processes as a Model of Neural Organization, American Journal of Psychology, 29.

[40]. Berman, P. W., Leibowitz H. W. (1965) Some Effects of Contour on Simultaneous Brightness Contrast, Journal of Experimental Psychology, 69, 251-256.

[41]. Gilmartin, Patricia. P.(1981) Influences of Map Context On Circle Perception, Annals, Association of American Geographers, $71: 2$, 253-258.

[42]. Luke, J. T. (1996) The Munsell Colour System: A Language for Colour, Fairchild Publications, New York. 
[43]. Gibson, J. J. (1950) The Perception of the Visual World, Houghten Mifflin Co. Boston.

[44]. Thorndyke, P. W. and Stasz. C. (1980) Individual Differences is Procedures for Knowledge Acquisition From Maps, Cognitive Psychology, 12, 137-175.

[45]. Munsell, A. H. (1991) A Colour Notation 17 ed., Munsell colour, Baltimore.

[46]. Katz, D. (1958) The Modes of Appearance of colours, in Readings in Perception. D. C. Beardslee and M. Wertheimer (eds.)Von Nostrand, Princeton

[47]. Koffka, K. (1935) Points and Lines as Stimuli, in Readings in perception, D. C. Beardslee and M. Wertheimer (eds.) (1958), Von Nostrand, Princeton.

[48]. Rubin, E. (1958) Figure and Ground, in Readings in Perception, D. C. Beardslee and M. Wertheimer (eds.) Von Nostrand, Princeton.

[49]. Oyama, T. (1960) Figure-Ground Dominance as a Function of Sector Angle, Brightness Hue and Orientation, Journal of Experimental Psychology, 60:5, 299-305.

[50]. Lindauer, M., Lindauer, J.(1970) Brightness Differences and the perception of Figure Ground Journal of Experimental Psychology, $84: 2,291-95$.

[51]. Wood, M. (1968) Visual Perception and Map Design, Cartographic Journal, 5, 54-64.

[52]. Spiess E. (1978) Some Graphic Means to Establish Visual Levels in Map Design. International Cartographic Association

[53]. McCleary, G. (1981) How to Design an Effective Graphics Presentation, Harvard Library of Computer Graphics, 1981 Mapping Collection, 17, P.A. Moore (ed.) 15-64.

[54]. MacEachren, A.M. and Mistrick, T. A. (1992) The Role of Brightness differences in Figure-ground: Is Darker Figure, The Cartographic Journal, 29, pp. 91-100

[55]. Keates, J. S. (1982) Understanding Maps, John Wiley and Sons, New York

[56]. Melara and Polts et.al. (1993) Primacy of Dimensions in Colour Perception, Journal of Experimental Psychology: Human Perception and Performance, 8:2, 194-214.

[57]. Eriksen, C. W. and Hake, H. W. (1955) Multidimensional Stimulus Differences and Accuracy of Discrimination, Journal of Experimental Psychology, 50, 153-160.

[58]. Mersey, Janet E. (1990) Colour and Thematic Map Design: The Role of Colour Scheme and Map Complexity in Choropleth Map Communication, Cartographica, 27:3

[59]. Wurm, Lee H., Legge, G. E., Isenberg, Lisa M. and Luebker, Andrew (1993) Colour Improves Object Recognition in Normal and Low Vision, Journal of Experimental Psychology: Human Perception and Performance, 19:4, 899-911.

[60]. Yantis, S., Johnston J.C. (1990) On the Locus of Visual Selection: Evidence from Focused Attention Tasks. Journal of Experimental Psychology: Human Perception and Performance, 16:1,135-149.

[61]. Robinson, A. H., Randall D. Sale, R. D., Morrison, J. and Muehrcke, Phillip, C. (1984) Element of Cartography, John Wiley, New York.

[62]. Ward Thomas, B. (1983) Response Tempo and Separable Integral Responding: Evidence for an Integral - to - separable Processing Sequence in Visual Perception, Journal of Experimental Psychology: Human Perception and Performance, 9:1, 103-112.

[63]. Imai and Garner (1965) Discriminability and Preference for Attributes in Free and Constrained Classification, Journal of Experimental Psychology, 69, 596-608.

[64]. Troscianko. T., Montagnos R., Leclerc J., and Malbert, E. et. al. (1991) The Role of Colour as a Monocular Depth Cue, Vision Research, 31:11, 1923-1930. 